\title{
Clinical and Dermoscopic Features of Fungal Melanonychia: Differentiating from Subungual Melanoma
}

\author{
Hak-Jun Kim ${ }^{1,2}$, Tae-Wook Kim ${ }^{1,2}$, Sung-Min Park ${ }^{1,2}$, Hyun-Joo Lee ${ }^{1}$, Gun-Wook Kim ${ }^{1}$, \\ Hoon-Soo Kim ${ }^{1}$, Byung-Soo Kim ${ }^{1}$, Moon-Bum Kim ${ }^{1}$, Hyun-Chang Ko, \\ ${ }^{1}$ Department of Dermatology, Pusan National University Hospital, Busan, ${ }^{2}$ Department of Dermatology, Pusan National University \\ Yangsan Hospital, Yangsan, Korea
}

Background: Fungal melanonychia (FM) is a rare nail disorder that presents as dark pigmentation in the nail plate because of fungal nail infection. The diagnosis of FM is occasionally confusing because its appearance is similar to melanonychia due to other causes including malignant melanoma. Dermoscopy could help increase the accuracy of diagnosing the cause of pigmented nail lesions. However, dermoscopic features of FM are not well elucidated. Objective: This study aimed to investigate clinical and dermoscopic characteristics of FM. Methods: The clinical features and dermoscopic findings of 20 patients diagnosed with FM and 14 patients diagnosed with subungual melanoma the Department of Dermatology of Pusan National University Hospitals (Busan and Yangsan) were retrospectively reviewed. Results: FM mainly occurred as a solitary form in the toenail. Patients in the FM group were older than those in the subungual melanoma group. The most distinguishable general dermoscopic features in FM were a distal diffuse pattern, distal linear pattern, and light brown to yellowish color. FM-associated specific dermoscopic patterns such as the reverse triangular pat-

Received June 3, 2019, Revised May 22, 2020, Accepted for publication May 22, 2020

Corresponding author: Hyun-Chang Ko, Department of Dermatology, Pusan National University Yangsan Hospital, 20 Geumo-ro, Mulgeum-eup, Yangsan 50612, Korea. Tel: 82-55-360-1678, Fax: 82-55-360-1679, E-mail: hcko@ pusan.ac.kr

ORCID: https://orcid.org/0000-0002-3459-5474

This is an Open Access article distributed under the terms of the Creative Commons Attribution Non-Commercial License (http://creativecommons. org/licenses/by-nc/4.0) which permits unrestricted non-commercial use, distribution, and reproduction in any medium, provided the original work is properly cited.

Copyright (c) The Korean Dermatological Association and The Korean Society for Investigative Dermatology tern, subungual hyperkeratosis, scale on the nail surface, and white or yellowish streaks were dominantly observed in the FM group compared to the subungual melanoma group. Conclusion: FM-associated dermoscopic patterns and distal diffuse and linear patterns could be helpful diagnostic clues for differential diagnosis of FM from subungual melanoma.

(Ann Dermatol 32(6) $460 \sim 465,2020$ )

\section{-Keywords-}

Dermoscopy, Fungal melanonychia, Subungual melanoma

\section{INTRODUCTION}

Fungal melanonychia (FM) is a rare nail disorder that presents with dark to brown pigmentation due to fungal infection of the nails. It is mainly caused by dematiaceous fungi and, rarely, by yeasts and non-dermatophyte fungi ${ }^{1}$. The diagnosis of FM is occasionally difficult because it may clinically mimic melanonychia due to other causes, such as melanocytic nevus and subungual melanoma. Recently, dermoscopy has been used to improve the accuracy of diagnosing the cause of pigmented nail lesions. A few studies have evaluated the dermoscopic features of $\mathrm{FM}^{2,3}$. However, these studies were limited by a small sample size or the absence of control groups. Here, we aimed to identify the clinical and dermoscopic characteristics of FM to enhance the accuracy of diagnosis. In this study, we have compared clinical and dermoscopic features of FM with those of subungual melanoma. 


\section{MATERIALS AND METHODS}

\section{Study population}

Of the patients with FM who visited the Department of Dermatology of Pusan National University Hospitals (Busan and Yangsan), South Korea from December 2014 to August 2018, a total of 20 patients who had dermoscopic records were included in this study. The diagnosis of FM was made based on clinical presentations and a mycological test. A potassium hydroxide $(\mathrm{KOH})$ smear test was performed in all patients, and only patients with positive test results were enrolled. Fungal culture was not always performed because of its low sensitivity and long incubation period. The control group comprised all patients diagnosed with subungual melanoma with dermoscopic records during the same period. Subungual melanoma was diagnosed by histopathological findings. Of the 14 patients who had subungual melanoma, 4 had invasive melanoma. All subungual melanomas were confirmed by a negative $\mathrm{KOH}$ smear test and excluded accompanying fungal infection. The study was exempted from the requirement for approval by the ethics committee of Pusan National University Yangsan Hospital (IRB no. 05-2019-057), and informed consent was obtained from the patients included in this study.

We received the patient's consent form about publishing all photographic materials.

\section{Method}

Data from the medical records of patients were used to investigate the clinical characteristics of the patients (age, sex, number of lesions, location, involved area, duration, and comorbidities). Clinical photographs were taken with a camera (EOS 50D; Canon, Tokyo, Japan). Dermoscopic images were acquired using a dermoscope (DermLite II PRO HR or DermLite II DL3; 3Gen, San Juan Capistrano, CA, USA) that was equipped with a digital camera (CyberShot DSC W-290; Sony Co, Tokyo, Japan).

All data were analyzed using IBM SPSS Statistics ver. 22.0 (IBM Corp., Armonk, NY, USA). p-values of less than 0.05 were considered significant.

\section{Evaluation of dermoscopic features}

General dermoscopic features (color and general shape) were investigated. The color of the nail lesion was divided into black, dark brown, light brown, yellow, gray, and red categories. The general shape of the melanonychia was classified as longitudinal, distal diffuse, proximal diffuse, distal linear, and total diffuse patterns (Fig. 1). FM-associated dermoscopic patterns, including reverse triangular shapes, subungual hyperkeratosis, scales on the nail surfaces, and yel-
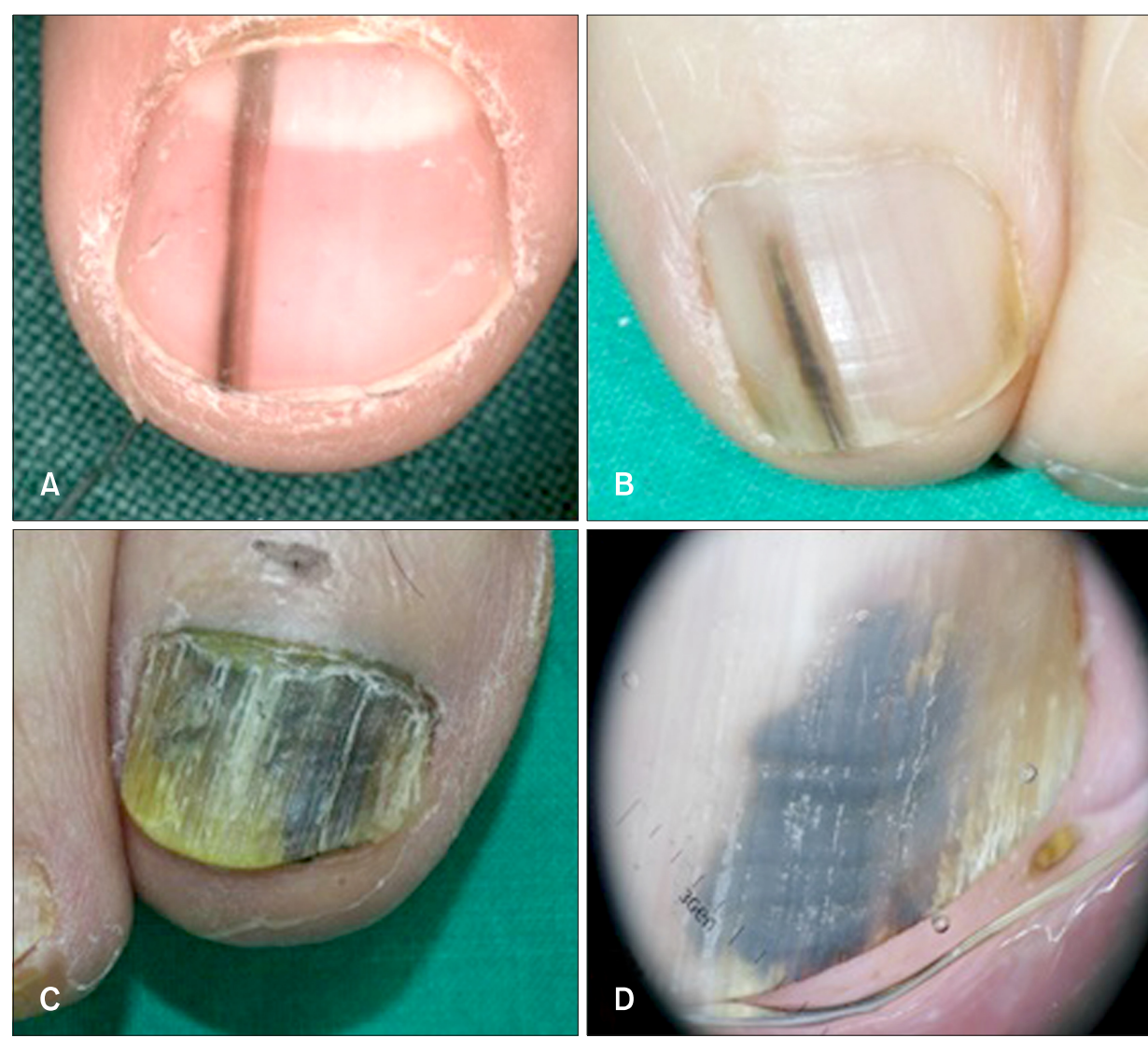

Fig. 1. The general shapes of melanonychia that were analyzed in this study; (A) longitudinal, (B) distal linear, $(C)$ total diffuse, and (D) distal diffuse pattern. 

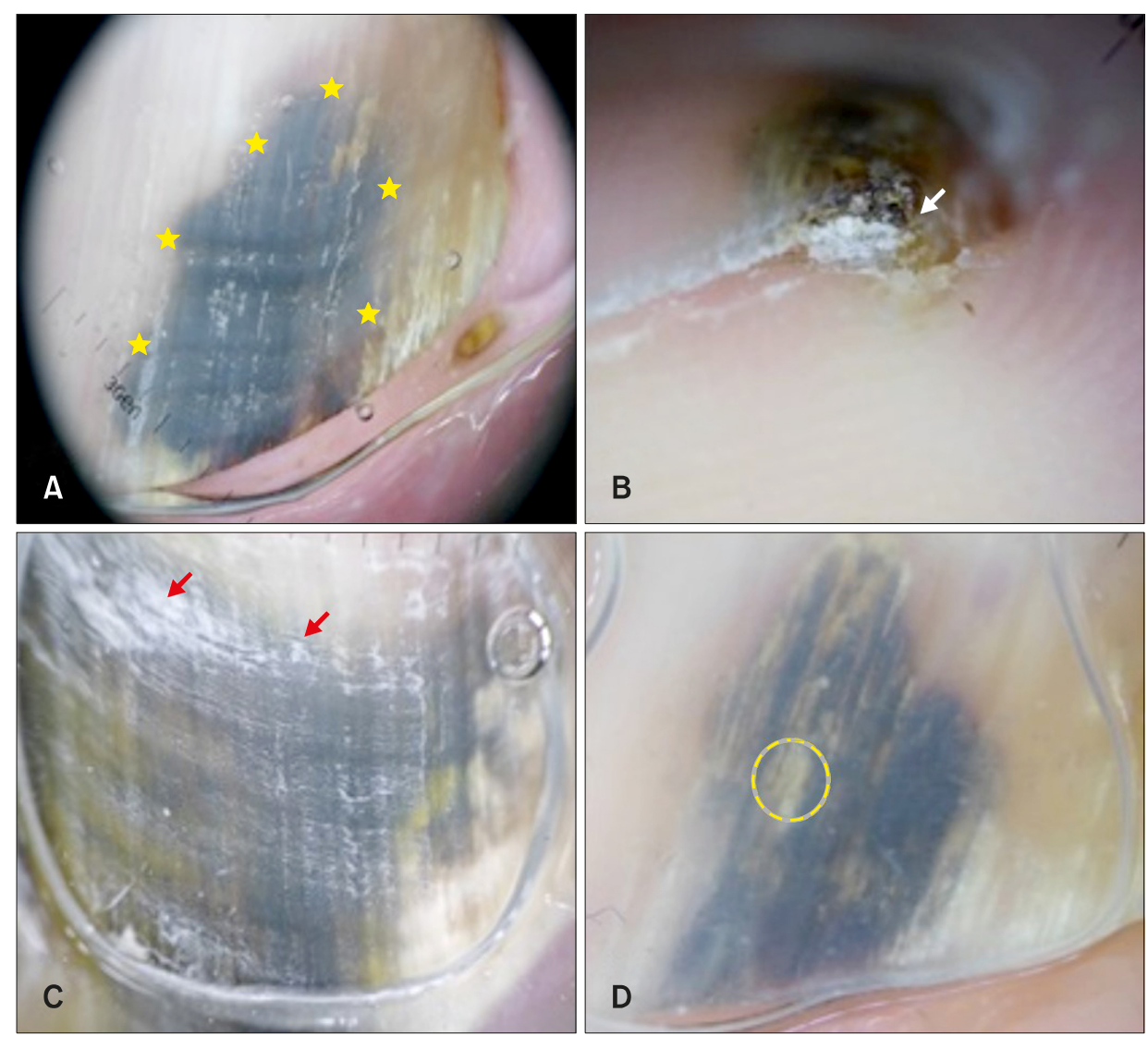

Fig. 2. The fungal melanonychiaassociated dermoscopic patterns. (A) Reverse triangular shape (yellow asterisks), (B) subungual hyperkeratosis (whitish arrow), (C) scales on the nail surface (red arrows), and (D) yellowish streaks (yellow circle).

lowish streaks, were evaluated (Fig. 2). Melanoma-associated patterns, such as Hutchinson's nail sign, irregularity, nail plate destruction, the triangular sign, and ulceration, were also evaluated to find a diagnostic clue that differentiates FM from subungual melanoma.

\section{RESULTS}

\section{Sociodemographic and clinical features}

The sociodemographic and clinical information of the FM and control groups (the subungual melanoma group) are presented in Table 1. We reviewed 34 patients (20 patients with FM and 14 patients with subungual melanoma). The mean age was 57.2 and 55.1 years in the FM and the subungual melanoma groups, respectively. The male-to-female ratio was $1: 1$ and 1:1.8 in the FM and subungual melanoma groups, respectively. FM occurred on 1.1 nail units per patient, and subungual melanoma occurred on 1.0 nail units per patient. The involved nail area of the FM group was smaller than that of the control group $(27.3 \%$ in $\mathrm{FM}, 38.7 \%$ in subungual melanoma, $p<0.05)$. There was no significant difference in incidence rates between the left side and the right side of the body. FM occurred in the toenails three times more often than in the
Table 1. Sociodemographic and clinical features of fungal melanonychia (FM group) and subungual melanoma (control group)

\begin{tabular}{lccc}
\hline \multicolumn{1}{c}{ Feature } & $\begin{array}{c}\text { FM group } \\
(\mathrm{n}=20)\end{array}$ & $\begin{array}{c}\text { Control group } \\
(\mathrm{n}=14)\end{array}$ & -value \\
\hline $\begin{array}{l}\text { Age of onset (yr) } \\
\text { Sex }\end{array}$ & $57.2(30 \sim 87)$ & $55.1(42 \sim 78)$ & $<0.05$ \\
$\quad$ Male & 10 & 6 & 0.062 \\
$\quad$ Female & 10 & 8 & \\
Location & & & \\
$\quad$ Finger:toe & $6: 16$ & $9: 5$ & $<0.05$ \\
$\quad$ Right:left & $11: 9$ & $8: 6$ & 0.133 \\
Mean no. of & 1.1 & 1.0 & 0.674 \\
nails involved & & & \\
Involved area (\%) & 27.3 & 38.7 & $<0.05$ \\
Duration (mo) & 18.2 & 20.8 & 0.088 \\
Comorbidity & & & \\
Hypertension & 9 & 8 & \\
Diabetes & 7 & 6 & \\
Dyslipidemia & 4 & 5 & \\
Cancer & 3 & 3 & \\
Others & 2 & 3 & \\
\hline
\end{tabular}

Values are presented as mean (range) or number only. 
Table 2. General dermoscopic features of fungal melanonychia (FM group) and subungual melanoma (control group)

\begin{tabular}{lccc}
\hline $\begin{array}{l}\text { General dermo- } \\
\text { scopic feature }\end{array}$ & $\begin{array}{c}\text { FM group } \\
(\mathrm{n}=20)\end{array}$ & $\begin{array}{c}\text { Control group } \\
(\mathrm{n}=14)\end{array}$ & $p$-value \\
\hline Color & & & \\
$\quad$ Black & $10(50.0)$ & $6(42.9)$ & 0.231 \\
Dark brown & $9(45.0)$ & $8(57.1)$ & 0.177 \\
Light brown & $9(45.0)$ & $0(0)$ & $<0.05$ \\
Yellow & $8(40.0)$ & $0(0)$ & $<0.05$ \\
Gray & $0(0)$ & $0(0)$ & - \\
Red & $0(0)$ & $0(0)$ & - \\
Multicolored & $13(65.0)$ & $11(78.6)$ & 0.159 \\
pattern & & & \\
(>2 colors $)$ & & & \\
General pattern & & & \\
Longitudinal & $6(30.0)$ & $6(42.9)$ & 0.056 \\
Distal diffuse & $7(35.0)$ & $0(0)$ & $<0.05$ \\
Proximal diffuse & $1(5.0)$ & $2(14.3)$ & 0.088 \\
Distal linear & $4(20.0)$ & $0(0)$ & $<0.05$ \\
Total diffuse & $2(10.0)$ & $5(35.7)$ & $<0.05$ \\
\hline
\end{tabular}

Values are presented as number (\%). -: not available.

fingernails. The most common onset site of FM was the first toe $(55.0 \%)$, followed by the first finger $(15.0 \%)$, and the second toe $(10.0 \%)$. In contrast, subungual melanoma nail lesions were found predominantly in the fingernails and mainly occurred in the second finger (35.7\%), first finger $(28.6 \%)$, and first toe $(14.3 \%)$. There was no significant difference in the disease duration between the two groups. Hypertension was the most common comorbidity in both groups, followed by diabetes and dyslipidemia.

\section{Dermoscopic features}

The general dermoscopic characteristics, such as color and shape of the melanonychia, are summarized in Table 2 . In the FM group, the most common color was black $(50.0 \%)$, followed by dark brown $(45.0 \%)$, light brown $(45.0 \%)$, and yellow $(40.0 \%)$. In the control group, the most common colors were dark brown (57.1\%) and black (42.9\%). Although black and dark brown were the predominant colors in both groups, the proportion of yellow melanonychia was significantly higher in the FM group than in the control group $(40.0 \%$ vs. $0 \%)$. The most common FM pattern was a distal diffuse type of melanonychia (35.0\%). Other common patterns included the longitudinal (30.0\%), distal linear $(20.0 \%)$, and total diffuse $(10.0 \%)$ types of melanonychia. Among these, the distal diffuse and linear patterns were more distinctive in the FM group than in the control group $(p<0.05)$. Statistical analyses between the FM and subungual melanoma groups were performed to identify specific dermoscopic differences (Table 3). We
Table 3. Specific dermoscopic features of fungal melanonychia (FM group) and subungual melanoma (control group)

\begin{tabular}{|c|c|c|c|}
\hline $\begin{array}{l}\text { Specific dermo- } \\
\text { scopic feature }\end{array}$ & $\begin{array}{l}\text { FM group } \\
(n=20)\end{array}$ & $\begin{array}{l}\text { Control group } \\
\qquad(n=14)\end{array}$ & $p$-value \\
\hline \multicolumn{4}{|c|}{ FM associated pattern } \\
\hline $\begin{array}{l}\text { Reverse } \\
\text { triangular shape }\end{array}$ & $10(50.0)$ & $0(0)$ & $<0.05$ \\
\hline $\begin{array}{l}\text { Subungual } \\
\text { hyperkeratosis }\end{array}$ & $7(35.0)$ & $2(14.3)$ & $<0.05$ \\
\hline $\begin{array}{l}\text { Scales on } \\
\text { the nail surface }\end{array}$ & $14(70.0)$ & $8(57.1)$ & $<0.05$ \\
\hline $\begin{array}{l}\text { White or } \\
\text { yellow streak }\end{array}$ & $18(90.0)$ & $0(0)$ & $<0.05$ \\
\hline \multicolumn{4}{|c|}{ MM associated pattern } \\
\hline Hutchinson sign & $0(0)$ & $8(57.1)$ & $<0.05$ \\
\hline $\begin{array}{l}\text { Pseudo-Hutchins } \\
\text { on sign }\end{array}$ & $0(0)$ & $4(28.6)$ & $<0.05$ \\
\hline $\begin{array}{l}\text { Nail plate } \\
\text { destruction }\end{array}$ & $5(25.0)$ & $4(28.6)$ & 0.312 \\
\hline Triangular sign & $0(0)$ & $5(35.7)$ & $<0.05$ \\
\hline Dots/globules & $4(20.0)$ & $7(50.0)$ & $<0.05$ \\
\hline Ulceration & $0(0)$ & $2(14.3)$ & $<0.05$ \\
\hline
\end{tabular}

Values are presented as number (\%). MM: malignant melanoma.

analyzed the following four FM-associated dermoscopic patterns: white or yellow streaks, reverse triangular patterns, subungual hyperkeratosis, and scales on the nail surfaces. In this study, we observed that all four features were positive predictors of FM (Fig. 2). In contrast, melanoma-associated patterns, such as Hutchinson's sign, pseudoHutchinson's sign, the triangular sign, nail plate destruction, dots/globules, and ulceration, were negative predictors of FM compared with the subungual melanoma $(p<0.05)$.

\section{DISCUSSION}

Melanonychia is defined by dark pigmentations in the nail unit. There are many different causes of melanonychia, ranging from nail matrix nevus, melanocytic activation due to drug use or trauma, subungual hemorrhage, and infection to malignant melanoma. Some of these causes have unique presentations, which make them easy to distinguish. However, most cases of melanonychia are difficult to distinguish because of their similar clinical presentations ${ }^{4,5}$.

FM is a rare nail disorder that presents with dark to brown pigmentation on the nail plate because of fungal nail infection. Some species of fungi can synthesize melanin. Unlike human melanin, which is derived from tyrosine, fungal melanin is synthesized via the pentaketide pathway. The fungal melanin serves as a barrier that protects the fungus from environmental stresses, such as temperature, 
radiation, and the host's immune system. In addition, melanin-synthesizing fungi are more resistant to ultraviolet and X-ray radiation and extreme temperatures compared to other fungi. Among the many species of fungi, the most frequently isolated fungi in FM are Trichophyton rubrum, a nondematiaceous dermatophyte, and Scytalidium dimidiatum, a dematiaceous fungi ${ }^{1,6}$. However, no analysis was made about causative organisms in this study because fungal culture was not routinely performed. Further studies will be needed to investigate characteristic patterns of FM based on specific causative pathogens.

We compared the clinical and dermoscopic features of FM with subungual melanoma to find diagnostic differences between the groups. The clinical features showed that the onset age of FM was relative older than that of the control. The mean age of FM was 57.2 years, which was significantly higher than that of individuals in the control group (55.1 years). FM showed a tendency to develop in a single nail unit (1.1 nail units per patients). However, some patients with a solitary FM lesion had multiple nail unit (mean 3.67) involvement of onychomycosis. In a study of 1897 Korean patients with onychomycosis, 31.3\% of patients showed multiple nail involvement (i.e., more than 5 nail units involved) ${ }^{7}$. In contrast, multiple nail involvement of FM was rarely observed in this study, with only one patient showing FM of multiple nails. This suggested that the incidence of FM is relatively low compared to onychomycosis. Furthermore, in previous studies, most cases of onychomycosis $(93.8 \%)$ involved toenails, and only $2.2 \%$ of the cases involved fingernails ${ }^{7}$. Similarly, FM occurred three times more often in toenails $(72.7 \%)$ than in fingernails $(27.2 \%)$ in this study. However, subungual melanoma was mainly observed in fingernails (64.3\%). To summarize the clinical analysis, FM tends to occur as a solitary lesion in the great toe, and its mean age of onset was higher than in cases of subungual melanoma.

Dermoscopy is a useful noninvasive tool that has been reported to improve diagnostic accuracy of nail pigmentation. Reports on the dermoscopic features of FM are scarce, and only a few original studies on FM have been reported ${ }^{2,8}$. Kilinc Karaarslan et al. ${ }^{3}$ have described the dermoscopic patterns in 14 cases of FM and suggested that multicolored pigmentation and reverse triangular patterns are distinctive features of FM. Hirata et al. ${ }^{9}$ have also reported a case of FM; they dermoscopically described it as a brown nail background with regular black lines. However, these studies were limited by a small sample size and the absence of a control group. Ohn et al. ${ }^{2}$ have reported the first comprehensive comparative analysis of dermoscopic findings between FM and other melanonychia caused by nail matrix nevus, melanocytic activation, and subungual melanoma.
Their study revealed that several dermoscopic features, such as reverse triangular patterns, subungual hyperkeratosis, and white or yellow streaks, were characteristics of FM. In this study, we observed that all these patterns were predominantly observed in FM cases. Among these patterns, white or yellow streaks and reverse triangular patterns were the most distinctive features of FM. Black pigmentation was the predominant color in the control group. This study also showed that the yellow color was a positive indicator for FM. Ohn et al. ${ }^{2}$ suggested that multicolored patterns were characteristic of FM. However, this study showed no significant difference in the presence of multicolored patterns between the FM and control groups. We chose cases of subungual melanoma as the control group because it usually has a heterogenous clinical presentation. The most common general pattern of dermoscopic findings in FM was the distal diffuse pattern (35.0\%), followed by the longitudinal $(30.0 \%)$ and distal linear $(20.0 \%)$ patterns. The two distal patterns were indicators of FM $(p<$ $0.05)$, but the longitudinal pattern was not. The longitudinal pattern was the predominant pattern in the control group (42.9\%). Two distal patterns that were not presented in the previous report were identified as characteristic FM dermoscopic findings in this study.

Piraccini et al. ${ }^{10}$ have reported that irregular white to yellow pigmentation and a "jagged edge" with sharp longitudinal whitish indentations are specific features of FM. In some previous studies, the spike or streak were useful indicators of fungal invasion of the nail plate. In this study, we used the term "white or yellow streak," and this pattern was more frequently observed in the FM group (90.0\%) than in the control group $(0 \%)$. The reverse triangular pattern is caused by fungal invasion from the distal nail plate $^{11}$, and our data showed that this pattern was prevalent in the FM group $(50.0 \%)$, but not in the melanoma group $(0 \%)$.

Subungual melanoma should always be included in the differential diagnosis of FM because melanoma is a lifethreatening disorder. FM could mimic subungual melanoma because it can present as a brown-black melanonychia with a heterogenous pattern ${ }^{12,13}$. We observed that melanoma-associated features aid in distinguishing between FM and subungual melanoma. The clinical diagnosis should be supported by dermoscopy findings as it may be beneficial if a biopsy or mycologic examination of the lesions is required. There are some limitations to this study. Since differential diagnosis of FM from malignant melanoma is important in cases of pigmented lesions of the nail units, especially in melanonychia of the elderly, histopathologic evaluation is essential for an accurate diagnosis. However, patients with FM in this study were diagnosed based on 
clinical findings and $\mathrm{KOH}$ smear tests only. This study has a retrospective design and a small number of patients; therefore, a prospective study with a larger scale is needed. However, this study is meaningful as it compares not only general dermoscopic patterns but also each specific dermoscopic feature between rare FM and subungual melanoma, which is the most important discriminating disease. In conclusion, we suggest that two general dermoscopic patterns (distal diffuse and linear) and FM-associated specific dermoscopic patterns can be used as diagnostic clue to differentiate FM from subungual melanoma. In the future, it is necessary to confirm the results through largescale follow-up studies.

\section{CONFLICTS OF INTEREST}

The authors have nothing to disclose.

\section{FUNDING SOURCE}

This work was supported by a 2-year Research Grant of Pusan National University.

\section{DATA SHARING STATEMENT}

The data that support the findings of this study are available from the corresponding author upon reasonable request.

\section{ORCID}

Hak-Jun Kim, https://orcid.org/0000-0003-1723-4244

Tae-Wook Kim, https://orcid.org/0000-0001-8922-8754

Sung-Min Park, https://orcid.org/0000-0002-4915-8111

Hyun-Joo Lee, https://orcid.org/0000-0002-1088-0975

Gun-Wook Kim, https://orcid.org/0000-0003-1599-7045

Hoon-Soo Kim, https://orcid.org/0000-0002-7649-1446

Byung-Soo Kim, https://orcid.org/0000-0003-0054-8570

Moon-Bum Kim, https://orcid.org/0000-0003-4837-0214

Hyun-Chang Ko, https://orcid.org/0000-0002-3459-5474

\section{REFERENCES}

1. Kim JA, Eun HC, Moon SE, Cho KH, Lee HS, Kim BS. Clinical features and classification of nail diseases. Korean J Dermatol 1999;37:1733-1743.

2. Ohn J, Choe YS, Park J, Mun JH. Dermoscopic patterns of fungal melanonychia: a comparative study with other causes of melanonychia. J Am Acad Dermatol 2017;76:488-493.e2.

3. Kilinc Karaarslan I, Acar A, Aytimur D, Akalin T, Ozdemir F. Dermoscopic features in fungal melanonychia. Clin Exp Dermatol 2015;40:271-278.

4. Jin H, Kim JM, Kim GW, Song M, Kim HS, Ko HC, et al. Diagnostic criteria for and clinical review of melanonychia in Korean patients. J Am Acad Dermatol 2016;74:1121-1127.

5. Kim JE, Park HJ, Lee JY, Cho BK. The compliance and long-term follow up of onychomycosis treatment. Korean J Med Mycol 2003;8:110-117.

6. Han MH, Choi JH, Sung KJ, Moon KC, Koh JK, Kim BC. Onychomycosis and Trichosporon beigelii. Korean J Dermatol 1999;37:1709-1715.

7. Hwang SM, Kim DM, Suh MK, Kwon KS, Kim KH, Ro BI, et al. Epidemiologic survey of onychomycosis in Koreans: multicenter study. Korean J Med Mycol 2011;16:35-43.

8. Tosti A, Schneider SL, Ramirez-Quizon MN, Zaiac M, Miteva M. Clinical, dermoscopic, and pathologic features of onychopapilloma: a review of 47 cases. J Am Acad Dermatol 2016;74:521-526.

9. Hirata SH, Yamada S, Enokihara MY, Di Chiacchio N, de Almeida FA, Enokihara MMSS, et al. Patterns of nail matrix and bed of longitudinal melanonychia by intraoperative dermatoscopy. J Am Acad Dermatol 2011;65:297-303.

10. Piraccini BM, Bruni F, Starace M. Dermoscopy of non-skin cancer nail disorders. Dermatol Ther 2012;25:594-602.

11. Lim SW, Suh MK, Ha GY. Clinical features and identification of etiologic agents in onychomycosis (1999-2002). Korean J Dermatol 2004;42:53-60.

12. Mun JH, Kim GW, Jwa SW, Song M, Kim HS, Ko HC, et al. Dermoscopy of subungual haemorrhage: its usefulness in differential diagnosis from nail-unit melanoma. Br J Dermatol 2013;168:1224-1229.

13. Duarte AF, Correia O, Barros AM, Ventura F, Haneke E. Nail melanoma in situ: clinical, dermoscopic, pathologic clues, and steps for minimally invasive treatment. Dermatol Surg 2015;41:59-68. 